\title{
CLASSES DE CHERN DES VARIÉTÉS SINGULIÈRES, REVISITÉES
}

\author{
PAOLO ALUFFI
}

\begin{abstract}
RÉSUMÉ. Nous introduisons une notion de groupe de proChow des variétés, qui reproduit la notion de groupe de Chow pour variétés complètes, et est fonctorielle par rapport aux morphismes arbitraires. Nous construisons une transformation naturelle du foncteur des fonctions constructibles vers le foncteur proChow, qui étend la transformation naturelle de MacPherson. Nous illustrons le résultat en donnant des démonstrations très courtes pour (des généralisations de) deux faits bien connus sur le classes de Chern-SchwartzMacPherson.
\end{abstract}

\section{INTRODUCTION}

Soit $X$ une variété sur un corps algébriquement clos de caractéristique nulle. Des notions équivalentes de classe de Chern totale de $X$ ont été données indépendamment par MarieHélène Schwartz ([9]) et par Robert MacPherson ([8]) pour les variétés algébriques complexes compactes, en homologie; la définition a été depuis étendue aux variétés algébriques complètes sur tous corps algébriquement clos de caractéristique nulle, dans le groupe de Chow $\mathrm{A}_{*} X$ de $X$. Nous appelons cette classe la classe de Chern-Schwartz-MacPherson (CSM) de $X$, dénotée $c_{\mathrm{SM}}(X)$.

La classe de CSM coïncide avec la classe de Chern ordinaire du fibré tangent quand $X$ est lisse : $c_{\mathrm{SM}}(X)=c(T X) \cap[X]$ dans ce cas. Elle satisfait également une propriété remarquable de fonctorialité : elle est définie comme la valeur $c_{*}\left(\mathbb{1}_{X}\right)$ prise par une transformation naturelle $c_{*} \mathrm{du}$ foncteur des fonctions constructibles $\mathrm{F}$ vers le foncteur de Chow $\mathrm{A}_{*}$, sur la fonction constante $\mathbb{1}_{X}$. Alexandre Grothendieck et Pierre Deligne avaient conjecturé l'existence de cette transformation naturelle; MacPherson la construit explicitement dans [8], en utilisant d'autres invariants importants qu'il introduit dans ce travail.

Dans cette note nous proposons une construction alternative des classes de Chern-SchwartzMacPherson, dans un groupe 'enrichi' de Chow $\widehat{\mathrm{A}}_{*} X$ (le groupe de proChow) obtenu en prenant des limites appropriées des groupes de Chow ordinaires. Le groupe de proChow est un foncteur covariant par rapport à tous les morphismes (tandis que le groupe de Chow ordinaire est fonctoriel seulement par rapport aux morphismes propres); nous démontrons que la transformation de proCSM correspondante $\mathrm{F} \leadsto \widehat{\mathrm{A}}_{*}$ est naturelle par rapport aux morphismes quelconques. Si $X$ est complète, le groupe de proChow de $X$ est canoniquement isomorphe au groupe de Chow ordinaire, et sa classe proCSM est égale à la classe de Chern-Schwartz-MacPherson.

Notre définition est directe, sans référence aux invariants auxiliaires comme les classes de Chern-Mather ou l'obstruction locale d'Euler. Pour illustrer son utilisation nous donnons des démontrations très condensées de deux résultats connus sur les classes de CSM, parus dans les C.R.A.S. : la formule du produit de Kwieciński ([7]), et la formule de EhlersBarthel-Brasselet-Fieseler pour les classes de CSM des variétés toriques ([3]). 


\section{Foncteur ProChow}

Nous travaillons sur un corps algébriquement clos $k$ de caractéristique nulle.

Soit $\mathscr{S}$ une catégorie de $k$-variétés. Pour $U$ dans $\mathscr{S}$, soit $\mathscr{S}_{U}$ la catégorie dont les objets sont les $\mathscr{S}$-morphismes $i: U \rightarrow Z^{i}$ de $U$ vers les variétés complètes dans $\mathscr{S}$, et les morphismes $j \mapsto i$ sont les diagrammes commutatifs de $k$-variétés

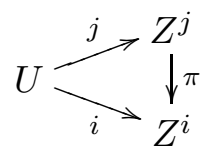

où $\pi$ est un morphisme propre. Nous supposons que les conditions suivantes sur $\mathscr{S}$ sont vérifiées :

- pour tout $U$ dans $\mathscr{S}$ et toute paire d'objets $i, j$ de $\mathscr{S}_{U}$, il y a un objet $k$ dans $\mathscr{S}_{U}$ tel que $k \rightarrow i$ et $k \rightarrow j$, et $k: U \hookrightarrow Z^{k}$ est une adhérence (c'est-à-dire, $k$ est un plongement ouvert, et $\left.\bar{U}=Z^{k}\right)$;

- si $U$ est lisse, on peut choisir l'adhérence $Z^{k}$ comme ci-dessus et bonne : c'est-à-dire, $Z^{k}$ est lisse, et le complément $Z^{k} \backslash U$ est un diviseur à croisements normaux et composantes lisses.

Par exemple, ces conditions sont satisfaites pour la catégorie de toutes les $k$-variétés (en caractéristique nulle, par résolution des singularités).

Définition 2.1. Le groupe de proChow de $U$ (par rapport a $\mathscr{S}$ ) est la limite $\widehat{\mathrm{A}}_{*}^{\mathscr{S}} U:=$ $\lim _{\leftarrow} \mathrm{A}_{*} Z^{i}$

Concrètement, un élément $\rho \in \widehat{\mathrm{A}}_{*}^{\mathscr{S}} U$ consiste en le choix d'un élément $\rho^{i}$ dans le groupe de Chow (conventionnel) $\mathrm{A}_{*} Z^{i}$ pour tout $i$ dans $\mathscr{S}_{U}$, sujet à la condition de compatibilité $\pi_{*} \rho^{j}=\rho^{i}$ pour tout $\pi: j \rightarrow i$. Nous disons que $\rho^{i}$ est la composante de $\rho$ dans $\mathrm{A}_{*} Z^{i}$.

Nous omettrons l'indice supérieur $\mathscr{S}$ quand aucune ambiguïté n'est probable; le lecteur notera que le groupe de proChow dépend de la catégorie choisie $\mathscr{S}$. Les faits suivants sont cependant indépendants de $\mathscr{S}$ (si $\mathscr{S}$ satisfait aux conditions de 'cofinalité' spécifiées ci-dessus) et immédiatement vérifiés :

Lemme 2.2. Avec les notations ci-dessus :

- Si $U$ est complète, il y a un isomorphisme canonique $\widehat{\mathrm{A}}_{*} U \cong \mathrm{A}_{*} U$.

- Pour spécifier un élément de $\widehat{\mathrm{A}}_{*} U$, il suffit de choisir un ensemble compatible de $\rho^{i} \in$ $\mathrm{A}_{*} Z^{i}$ pour toute adhérence $i: U \rightarrow Z^{i}$ dans $\mathscr{S}$.

- Si, en outre, $U$ est lisse, il suffit de choisir un ensemble compatible de $\rho^{i} \in \mathrm{A}_{*} Z^{i}$ pour toute bonne adhérence $i: U \rightarrow Z^{i}$ dans $\mathscr{S}$.

Chaque sous-schéma $B$ de $U$ détermine un élément distingué $[\bar{B}]$ de $\widehat{A}_{*} U$ : pour chaque adhérence $j: U \rightarrow Z^{j}$, on choisit la classe $[\bar{B}] \in \mathrm{A}_{*} Z^{j}$ de l'adhérence de $B$ dans $Z^{j}$; ce choix est clairement compatible. Si $U$ est complète, $[\bar{B}] \in \widehat{\mathrm{A}}_{*} U \cong \mathrm{A}_{*} U$ est la 'classe fondamentale' ordinaire de $\bar{B}$.

Le groupe de proChow $\widehat{\mathrm{A}}_{*}=\widehat{\mathrm{A}}_{*}^{\mathscr{S}}$ est un foncteur $\mathscr{S} \leadsto$ Groupes Abéliens : si $f: X \rightarrow Y$ est un morphisme dans $\mathscr{S}$, alors $j \rightarrow j \circ f$ induit un foncteur $\mathscr{S}_{Y} \rightarrow \mathscr{S}_{X}$, et donc un homomorphisme $f_{*}: \widehat{\mathrm{A}}_{*} X \rightarrow \widehat{\mathrm{A}}_{*} Y$. Concrètement, pour $\rho \in \widehat{\mathrm{A}}_{*} X$ et $j: Y \rightarrow Z^{j}$ dans $\mathscr{S}_{Y}$, la composante de $f_{*} \rho$ dans $\mathrm{A}_{*} Z^{j}$ est simplement égale à la composante de $\rho$. Si $f$ est propre et $X$ et $Y$ sont complètes, alors $f_{*}: \widehat{\mathrm{A}}_{*} X \cong \mathrm{A}_{*} X \rightarrow \mathrm{A}_{*} Y \cong \widehat{\mathrm{A}}_{*} Y$ est le push-forward propre ordinaire des groupes de Chow. On note cependant que tandis que $A_{*}$ est fonctoriel seulement par rapport aux morphismes propres, le proChow $\widehat{A}_{*}$ est fonctoriel par rapport à tous les morphismes dans $\mathscr{S}$. 


\section{Classes proCsM}

Avec $\mathscr{S}$ comme dans la Section 2, et $X$ dans $\mathscr{S}$, nous définissons le groupe des fonctions $\mathscr{S}$-constructibles $\mathrm{F}^{\mathscr{S}}(X)$ comme le groupe des combinaisons finies $\mathbb{Z}$-linéaires des fonctions caractéristiques $\mathbb{1}_{U}$ (où $\mathbb{1}_{U}(p)=1$ si $p \in U$, et 0 si $p \in X \backslash U$ ) où $U$ sont des sous-variétés localement fermées lisses de $X$, telles que les inclusions $U \subset X$ sont des morphismes de $\mathscr{S}$.

Nous posons maintenant une condition supplémentaire sur $\mathscr{S}$. Nous demandons que le push-forward conventionnel des fonctions constructibles (définies en prenant la caractéristique d'Euler fibre à fibre, voir [8] pour le cas complexe) préserve la $\mathscr{S}$-constructibilité : c'està-dire, qu'il définisse un push-forward $f_{*}: \mathrm{F}^{\mathscr{S}}(X) \rightarrow \mathrm{F}^{\mathscr{S}}(Y)$ pour chaque morphisme $f: X \rightarrow Y$ dans $\mathscr{S}$. Nous exigeons également que $\mathbb{1}_{X}$ soit $\mathscr{S}$-constructible pour chaque $X$ dans $\mathscr{S}$.

Sous ces conditions, $\mathrm{F}^{\mathscr{S}}$ définit (en caractéristique nulle!) un foncteur covariant $\mathscr{S} \leadsto$ Groupes Abéliens, et chaque $X$ dans $\mathscr{S}$ détermine un élément distingué $\mathbb{1}_{X} \in \mathrm{F}^{\mathscr{S}}(X)$. Nous omettrons habituellement l'indice supérieur $\mathscr{S}$.

Nous définissons maintenant un homomorphisme $\mathrm{F}(X) \rightarrow \widehat{\mathrm{A}}_{*} X, \alpha \mapsto\{\alpha\}$, et un élément distingué $\{X\}:=\left\{\mathbb{1}_{X}\right\} \in \widehat{\mathrm{A}}_{*} X$. Nous commençons par le cas lisse :

Définition 3.1. Soit $U$ lisse, dans $\mathscr{S}$. La classe proCSM de $U$ dans $\widehat{A}_{*} U$, notée $\{U\}$, est l'élément du groupe de proChow déterminé par $c\left(\Omega_{\bar{U}}(\log D)^{\vee}\right) \cap[\bar{U}] \in \mathrm{A}_{*} \bar{U}$ pour toute bonne adhérence $\bar{U}$ de $U$ dans $\mathscr{S}_{U}$, où $D=\bar{U} \backslash U$ est le diviseur à croisements normaux correspondant, et $\Omega \frac{1}{U}(\log D)^{\vee}$ désigne le dual du fibré de formes différentielles avec pôles logarithmiques le long de $D$.

Ce choix est compatible selon la section 2, comme on le démontrera dans le Théorème 3.3 ; donc il définit un élément de $\widehat{\mathrm{A}}_{*} U$, d'après le Lemme 2.2 .

Soit maintenant $X$ une variété arbitraire (c'est-á-dire, pas nécessairement lisse) dans $\mathscr{S}$, et soit $\alpha \in F(X)$ une fonction constructible sur $X$. Soit $\alpha=\sum_{U} m_{U} \mathbb{1}_{U}$, avec $U$ lisse, localement fermée, $i_{U}: U \subset X$ dans $\mathscr{S}$, et $m_{U} \in \mathbb{Z}$.

Définition 3.2. La classe proCSM de $\alpha$ est la somme $\{\alpha\}=\sum_{U} m_{U} i_{U *}\{U\} \in \widehat{\mathrm{A}}_{*} X$. La classe proCSM de $X$ est la classe $\{X\}:=\left\{\mathbb{1}_{X}\right\}$.

Nous pouvons maintenant énoncer et démontrer le résultat principal de cette note.

Théorème 3.3. Avec ces notations :

(1) Les choix donnés dans la Définition 3.1 sont compatibles : c'est-à-dire, si $i: U \rightarrow$ $\bar{U}^{i}$ et $j: U \rightarrow \bar{U}^{j}$ sont des bonnes adhérences de $U$ dans $\mathscr{S}_{U}$, de compléments $D^{i}, D^{j}$, et $\pi: \bar{U}^{j} \rightarrow \bar{U}^{i}$ est un morphisme propre tels que $i=\pi \circ j$, alors $\pi_{*}\left(c\left(\Omega_{\bar{U}^{j}}^{1}\left(\log D^{j}\right)^{\vee}\right) \cap\left[\bar{U}^{j}\right]\right)=c\left(\Omega_{\bar{U}^{i}}^{1}\left(\log D^{i}\right)^{\vee}\right) \cap\left[\bar{U}^{i}\right]$.

(2) La Définition 3.2 est indépendante des choix : c'est-à-dire, si $\alpha=\sum_{U} m_{U} \mathbb{1}_{U}=$ $\sum_{V} n_{V} \mathbb{1}_{V}$ sont deux manières d'exprimer $\alpha$ comme combinaison linéaire finie des fonctions caractéristiques des sous-variétés localement fermées lisses de $X$, alors $\sum_{U} m_{U} i_{U *}\{U\}=\sum_{V} n_{V} i_{V *}\{V\}$.

(3) L'homomorphisme $\mathrm{F}(X) \rightarrow \widehat{\mathrm{A}}_{*} X, \alpha \mapsto\{\alpha\}$ donné dans la Définition 3.2 définit une transformation naturelle $\mathrm{F} \leadsto \widehat{\mathrm{A}}_{*}$; c'est-à-dire, $f_{*}\{\alpha\}=\left\{f_{*}(\alpha)\right\}$ pour chaque morphisme $f: X \rightarrow Y$ in $\mathscr{S}$.

(4) Si $X$ est complète, alors la classe proCSM de $X$ est la classe de Chern-SchwartzMacPherson conventionnelle $:\{X\}=c_{\mathrm{SM}}(X) \in \mathrm{A}_{*} X \cong \widehat{\mathrm{A}}_{*} X$. 
On peut démontrer le trois premiers points indépendamment du résultat de MacPherson en [8]; cela est fait dans [1]. Dans le cas particulier des variétés complètes et des fonctions propres, le troisième point donne une transformation naturelle comme prescrite par la (version Chow de la) conjecture de Grothendieck-Deligne; l'égalité des classes proCSM et des classes CSM pour les variétés complètes découle alors de l'unicité de cette transformation naturelle (qui est une conséquence immédiate de la résolution des singularités).

Nous présentons ici une preuve qui utilise le théorème de MacPherson (c'est-à-dire, le fait que la transformation de MacPherson $c_{*}$ est naturelle), ce qui permet un raisonnement plus rapide.

Démonstration. - Si $U$ est lisse, $\bar{U}$ est une bonne adhérence de $U$, et $D=\bar{U} \backslash U$, alors

$$
c\left(\Omega_{\bar{U}}(\log D)^{\vee}\right) \cap[\bar{U}]=c_{*}\left(\mathbb{1}_{U}\right) \in A_{*} \bar{U} .
$$

Ceci découle facilement du fait que $c_{*}$ est naturel et d'un calcul explicite de classes de Chern ; voir par exemple la Proposition 15.3 dans [6], ou le Théorème 1 dans [2].

(1) découle de $(\dagger)$, du fait que $c_{*}$ est naturel, et de la définition de push-forward des fonctions constructibles :

$\pi_{*}\left(c\left(\Omega_{\bar{U}^{i}}^{1}\left(\log D^{i}\right)^{\vee}\right) \cap\left[\bar{U}^{i}\right]\right)=\pi_{*} c_{*}\left(\mathbb{1}_{U}\right)=c_{*} \pi_{*}\left(\mathbb{1}_{U}\right)=c_{*}\left(\mathbb{1}_{U}\right)=c\left(\Omega_{\bar{U}^{j}}^{1}\left(\log D^{j}\right)^{\vee}\right) \cap\left[\bar{U}^{j}\right]$.

La preuve des autres points est simplifiée par la version alternative suivante de la Définition 3.2 :

Lemme 3.4. Pour chaque $z: X \rightarrow Z$ dans $\mathscr{S}_{X}$, et chaque $\alpha \in \mathrm{F}(X)$, la composante de $\{\alpha\}$ dans $\mathrm{A}_{*} Z$ est $c_{*}\left(z_{*}(\alpha)\right)$.

Pour démontrer le lemme, on utilise $(\dagger)$ pour écrire la composante de $\{U\}$ dans $A_{*} Z$, pour $U$ lisse et $z_{U}: U \rightarrow Z$ dans $\mathscr{S}$, comme $c_{*}\left(z_{U *}\left(\mathbb{1}_{U}\right)\right)$. Si $\alpha \in \mathrm{F}(X)$, alors $\alpha=\sum_{U} m_{U} \mathbb{1}_{U}$, avec $U$ lisse et l'inclusion $U \subset X$ dans $\mathscr{S}$; donc, pour chaque $z: X \rightarrow Z$, la composante de $\sum_{U} m_{U}\{U\}$ dans $\mathrm{A}_{*} Z$ est $\sum_{U} m_{U} c_{*}\left(z_{*} \mathbb{1}_{U}\right)=c_{*}\left(z_{*}\left(\sum_{U} m_{U} \mathbb{1}_{U}\right)\right)=c_{*}\left(z_{*}(\alpha)\right)$, comme énoncé.

(2) s'ensuit immédiatement, puisque $c_{*}\left(z_{*}(\alpha)\right)$ dépend seulement de $\alpha$, et pas de la décomposition $\alpha=\sum_{*} m_{U} \mathbb{1}_{U}$. D'ailleurs, si $X$ est elle-même complète, alors en prenant $Z=X, z=\operatorname{id}_{X}$, et $\alpha=\mathbb{1}_{X}$ dans le Lemme 3.4, on trouve (4).

Enfin, le Lemme 3.4 implique (3). En effet, soit $z: Y \rightarrow Z$ un objet quelconque de $\mathscr{S}_{Y}$; alors $w \circ f$ est un objet de $\mathscr{S}_{X}$ et, d'après la définition de push-forward de groupes de proChow, la composante dans $\mathrm{A}_{*} Z$ du push-forward $f_{*}\{\alpha\}$ est simplement égale à la composante de $\{\alpha\}$ dans $\mathrm{A}_{*} Z$. D'après le Lemme 3.4, cette composante est $c_{*}\left((w \circ f)_{*} \alpha\right)=$ $c_{*}\left(z_{*}\left(f_{*}(\alpha)\right)\right)$, et encore d'après le Lemme 3.4 elle est égale à la composante de $\left\{f_{*}(\alpha)\right\}$ dans $\mathrm{A}_{*} Z$, ce qui prouve (3).

\section{EXEMPLES}

Nous illustrons le formalisme présenté dans $\S 2$ et $\S 3$ en donnant des preuves très courtes (et valides dans le cadre proCSM) de deux résultats connus sur les classes de ChernSchwartz-MacPherson.

Nous utiliserons des catégories différentes, comme permis par les constructions données dans les sections précédentes. En notant par $\widehat{\mathrm{A}}_{*}$ le foncteur proChow obtenu à partir de $\mathscr{S}=$ la catégorie de toutes $k$-variétés, et par $\mathrm{F}$ le foncteur de fonctions constructibles sur cette catégorie, on note que pour toute autre catégorie $\mathscr{S}$ on a des homomorphismes canoniques $\mathrm{F}^{\mathscr{S}}(X) \rightarrow \mathrm{F}(X), \widehat{\mathrm{A}}_{*}(X) \rightarrow \widehat{\mathrm{A}}_{*}^{\mathscr{S}}(X)$, compatibles avec les transformations naturelles proCSM correspondantes. 
Le premier résultat est la formule de produit de Michał Kwieciński. On prend pour $\mathscr{S}$ la catégorie de produits $X \times Y$ (techniquement, de paires $(X, Y)$ ), où $X$ et $Y$ sont des $k$-variétés, et dont les morphismes $X_{1} \times Y_{1} \rightarrow X_{2} \times Y_{2}$ consistent en des paires $(f, g)$, où $f: X_{1} \rightarrow X_{2}$ et $g: Y_{1} \rightarrow Y_{2}$ sont morphismes. Les conditions détaillées dans $\S 2$ et $\S 3$ sont clairement vérifiées (en caractéristique nulle), et on a donc un foncteur $\widehat{A}_{*}^{\times}$de proChow et un foncteur $\mathrm{F}^{\times}$de fonctions $\mathscr{S}$-constructibles. Le groupe $\mathrm{F}^{\times}(X \times Y)$ consiste en des fonctions $\alpha \otimes \beta$ définies par $\alpha \otimes \beta(x, y)=\alpha(x) \beta(y)$, où $\alpha \in \mathrm{F}(X), \beta \in \mathrm{F}(Y)$ sont des fonctions constructibles (ordinaires). On note la classe proCSM correspondante par $\{\alpha \otimes \beta\}^{\times}$.

On voit en outre qu'il y a un homomorphisme canonique évident

$$
\widehat{\mathrm{A}}_{*}(X) \otimes \widehat{\mathrm{A}}_{*}(Y) \stackrel{\otimes}{\longrightarrow} \widehat{\mathrm{A}}_{*}^{\times}(X \times Y),
$$

$(\alpha, \beta) \mapsto \alpha \otimes \beta$, induit par les produits extérieurs pour les groupes de Chow ordinaires ([4], $\S 1.10)$.

Théorème 4.1. Soient $X$ et $Y$ deux variétés, $\alpha \in \mathrm{F}(X), \beta \in \mathrm{F}(Y)$ et $\alpha \otimes \beta \in \mathrm{F}^{\times}(X \times Y)$ comme ci-dessus. Alors $\{\alpha \otimes \beta\}^{\times}=\{\alpha\} \otimes\{\beta\}$.

Démonstration. - Par bilinéarité, l'énoncé suit du cas $\alpha=\mathbb{1}_{U}, \beta=\mathbb{1}_{V}$ pour $U, V$ sous-variétés lisses de $X, Y$ resp.; c'est-à-dire qu'il suffit de vérifier que pour $U, V$ lisses, et pour $\bar{U}, \bar{V}$ bonnes adhérences of $U, V$, de compléments $D=\bar{U} \backslash U, E=\bar{V} \backslash V$,

$$
c\left(\Omega \frac{1}{\bar{U} \times \bar{V}}(\log (D+E))^{\vee}\right) \cap[\bar{U} \times \bar{V}]=\left(c\left(\Omega \frac{1}{U}(\log D)^{\vee}\right) \cap[\bar{U}]\right) \otimes\left(c\left(\Omega \frac{1}{V}(\log E)^{\vee}\right) \cap[\bar{V}]\right),
$$

et ceci suit immédiatement du calcul standard des classes de Chern pour le fibré de formes différentielles avec pôles logarithmiques.

Le cas particulier dans lequel $X$ et $Y$ sont complètes reproduit le théorème de Kwieciński ([7]), puisque dans ce cas-là tous les groupes de proChow dans l'énoncé sont isomorphes aux groupes conventionnels de Chow (d'après le Lemme 2.2), et les classes proCSM sont égales aux classes de Chern-Schwartz-MacPherson (d'après le Théorème 3.3).

Notre deuxième exemple est la formule de Fritz Ehlers pour la classe de Chern-SchwartzMacPherson d'une variété torique; voir [5], p. 113, et [3] pour la preuve dans le cadre CSM conventionnel. Pour donner un énoncé et une preuve dans le cas plus général de proChow, soit $\mathscr{S}$ la catégorie de $k$-variétés toriques, avec les morphismes $T$-équivariants. Le foncteur et les classes proCSM correspondants seront notés respectivement $\widehat{A}^{\top}$ et $\{X\}^{\top}$; la classe fondamentale de $B \subset X$ dans $\widehat{A}_{*}^{\top}(X)$ sera notée $[\bar{B}]$.

Théorème 4.2. Soit $X$ une variété torique. Alors $\{X\}^{\top}=\sum_{B \in X / T}[\bar{B}] \in \widehat{A}_{*}^{\top}(X)$, où la somme porte sur l'ensemble (fini) des T-orbites.

Démonstration. - Puisque $X$ est l'union des $T$-orbites $B$ nous avons $\{X\}^{\top}=\sum\{B\}^{\top}$, et par conséquent il suffit de montrer que si $B$ est l'orbite ouverte dans la sous-variété torique $\bar{B} \subset X$ alors $\{B\}^{\top}=[\bar{B}] \in \widehat{\mathrm{A}}_{*}^{\top}(B)$; ce qui est équivalent à montrer que si $\bar{B}$ est une bonne adhérence (torique) de $B$, et $D=\bar{B} \backslash B, c\left(\Omega \frac{1}{B}(\log D)^{\vee}\right) \cap[\bar{B}]=[\bar{B}] \in \mathrm{A}_{*}(\bar{B})$ : et ceci est vrai puisque $\Omega \frac{1}{B}(\log D)$ est trivial ([5], Proposition, p. 87).

Dans le cas particulier où $X$ est une variété torique complète, on retrouve la formule d'Ehlers.

Le théorème 4.2 admet (avec la même preuve) une généralisation aux plongements toroïdaux qui ne sont pas nécessairement normaux. 


\section{RÉFÉRENCES}

[1] P. Aluffi. Limits of Chow groups, and a new construction of Chern-Schwartz-MacPherson classes. FSU0514, arXiv :math.AG/0507029.

[2] P. Aluffi. Differential forms with logarithmic poles and Chern-Schwartz-MacPherson classes of singular varieties. C. R. Acad. Sci. Paris Sér. I Math., 329(7) : 619-624, 1999.

[3] G. Barthel, J.-P. Brasselet, K.-H. Fieseler. Classes de Chern des variétés toriques singulières. C. R. Acad. Sci. Paris Sér. I Math., 315(2) : 187-192, 1992.

[4] W. Fulton. Intersection theory. Springer-Verlag, Berlin, 1984.

[5] W. Fulton. Introduction to toric varieties, volume 131 of Annals of Mathematics Studies. Princeton University Press, Princeton, NJ, 1993. The William H. Roever Lectures in Geometry.

[6] M. Goresky, W. Pardon. Chern classes of automorphic vector bundles. Invent. Math., 147(3) : 561-612, 2002.

[7] M. Kwieciński. Formule du produit pour les classes caractéristiques de Chern-Schwartz-MacPherson et homologie d'intersection. C. R. Acad. Sci. Paris Sér. I Math., 314(8) : 625-628, 1992.

[8] R. D. MacPherson. Chern classes for singular algebraic varieties. Ann. of Math. (2), 100 : 423-432, 1974.

[9] M.-H. Schwartz. Classes caractéristiques définies par une stratification d'une variété analytique complexe. C. R. Acad. Sci. Paris, 260 : 3262-3264, 3535-3537, 1965.

Dept. of Mathematics, Florida State University, Tallahassee FL 32306, U.S.A.

Max-Planck-Institut für Mathematik, Postfach 7280, D-53072 Bonn, Deutschland

E-mail address: aluffi@math.fsu.edu 